Block and deliver

Angew. Chem. Int. Ed. http://doi.org/

fz5qc7 (2012)

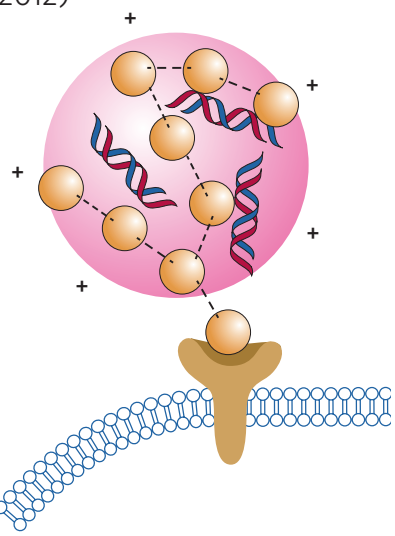

The transmembrane G-protein receptor CXCR4 and its ligand CXCL12 are known to be involved in the metastasis of cancer cells to other sites in the body; therefore, blocking this interaction is an important strategy in cancer therapy. Small-molecule inhibitors of CXCR4, for example, the bicyclam derivative AMD3100, have shown antagonistic behaviour and antimetastatic activity in some cancers. Now, David Oupický and colleagues have synthesized a polycationic biodegradable system incorporating AMD3100 and plasmid DNA, which demonstrates both CXCR4 antagonism and gene delivery to bone cancer cells. These polycation-DNA species, or polyplexes, show the concurrent ability to inhibit cancer cell invasion, deliver plasmid DNA and mediate transfection. The polyplexes are made by the Michael addition polymerization of AMD3100 with a disulphide-containing bisacrylamide, followed by addition of plasmid DNA. Although the polyplexes do not need to be internalized to show CXCR4 antagonism, this is required for the gene delivery step. Oupický and colleagues found that a different uptake pathway other than the CXCR4 receptor is used for polyplex transfection and delivery. AS

\section{Understanding endocytosis Cell 150, 508-520 (2012)}

Clathrin-mediated endocytosis - a process by which cells engulf extracellular molecules and that involves membrane invaginations coated with the protein clathrin - has long been investigated. However, how clathrin and other endocytic proteins reshape the plasma membrane into a highly curved, budding configuration that leads to the formation of long invaginations, and how these break off to become vesicles, have remained unclear. Using both fluorescence and electron microscopy, Wanda Kukulski and colleagues reconstructed the three-dimensional structure of more than 200 endocytic intermediates in yeast cells. The researchers show that actin polymerization, and not the proteins present at the endocytic site, begins to induce membrane curvature and the formation of an invagination tip, where the proteins arrange to form a coat. They also show that amphiphysin proteins stabilize the growing invagination into a tubule before neck formation and scission occurs. On average, the process takes $9 \mathrm{~s}$ and the invagination depth reaches $100 \mathrm{~nm}$. The wealth of data provided by the study should trigger further understanding of how proteins dynamically shape membranes.

\section{Hybrid electrolytes}

Adv. Mater. http://doi.org/fz5hpc (2012)

Compared with lithium-ion batteries, secondary lithium-metal batteries demonstrate enhanced energy-storage capabilities due to the high specific capacity of metallic lithium and the possibility of using non-lithiated compounds as cathodes. And although ionic liquids used as electrolytes have proved successful for controlling nucleation and growth of lithium dendrites they still suffer from high costs, poor mechanical properties and low conductivities. Lynden Archer and colleagues now show that

\title{
Plasmonics making waves
}

Surface plasmons play a key role in nanophotonics as they enable light manipulation at subwavelength scales. Active control of surface plasmon properties of a device is, however, more difficult to achieve. One of the mechanisms studied to achieve this control are structural phase changes of the active material, such as solid to liquid transitions. S.R. C. Vivekchand and colleagues have now demonstrated an advantage of this strategy by observing that light can couple to surface plasmons better for a liquid metal than for a solid. The metal studied, gallium, has a relatively low melting point of just below $303 \mathrm{~K}$. This makes it easy to compare the optical properties of grating structures for liquid and solidified structures. The liquid phase showed a much stronger light absorption at plasmon resonances. This is because of a broadening of the electronic band structure in the liquid phase that reduces losses for the surface plasmons. The reflective properties can therefore be efficiently controlled simply by heating and cooling the device.

ionic-liquid-tethered nanoparticle hybrid electrolytes consisting of silica nanoparticles densely grafted with imidazolium-based ionic liquid chains can retard lithium dendrite growth in rechargeable batteries with metallic lithium anodes. The brush-like structure of the ionic liquid molecules tethered to the particles is believed to facilitate the dispersion of silica nanoparticles, and may act as a reservoir of anions thus reducing anion inhomogeneities in the electrolyte. The electrolytes are demonstrated in full-cell studies using both high-energy $\mathrm{Li} / \mathrm{MoS}_{2}$ and high-power $\mathrm{Li} / \mathrm{TiO}_{2}$ secondary batteries, and display enhanced electrochemical and interfacial stability together with improved thermal stability compared with propylene carbonate electrolytes.

Up and away

Appl. Phys. Lett. 101, 023302 (2012)

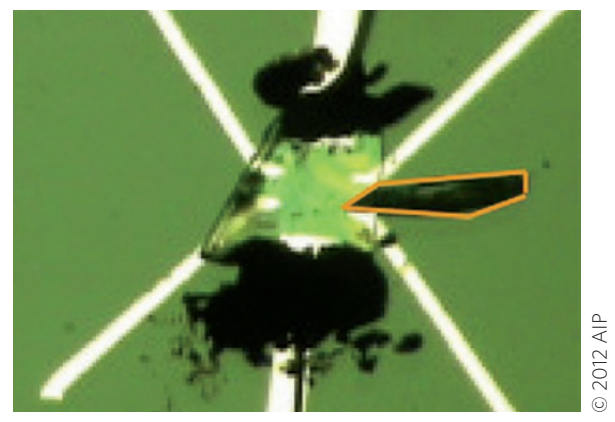

Tetrathiofulvalene (TTF) and 7,7,8,8tetracyanoquinodimethane (TCNQ) form a well-known charge-transfer complex, in which an electron from the highest occupied molecular orbital of TTF is transferred to TCNQ. In TTF-TCNQ crystals, this charge transfer leads to metallic conduction, which is unusual for organic materials. Only recently has it been shown that such charge transfer also occurs when two single crystals of the molecules are laminated onto each other, and that this results in the formation of a conducting interface. The details of such interfaces are yet to be explored, and Bertram Batlogg and co-workers now elucidate the microscopic processes that contribute to their formation. The researchers demonstrate that, when two crystals of TTF and TCNQ are placed next to each other at room temperature, sublimation and redeposition of TTF molecules can induce a conducting surface layer on TCNQ, albeit very slowly. They suggest that local sublimation facilitates the formation of conductive interfaces in laminated crystals, and show that cooling slows down the process.

Written by Vincent J. Dusastre, Joerg Heber, Christian Martin, Pep Pàmies and Alison Stoddart. 